\title{
Efficient Multihop Broadcast for Wideband Systems
}

\author{
Ivana Maric \\ WINLAB, Rutgers University \\ ivanam@winlab.rutgers.edu
}

\author{
Roy Yates \\ WINLAB, Rutgers University \\ ryates@winlab.rutgers.edu
}

\begin{abstract}
In this paper we address the minimum-energy broadcast problem. To increase the energy efficiency, we allow nodes that are out of the transmission range of a transmitter to collect the energy of unreliably received overheard signals. As a message is forwarded through the network, a node will have multiple opportunities to reliably receive the message by collecting energy during each retransmission. We refer to this strategy as accumulative broadcast. Under the assumption that the nodes reliably forward messages, we formulate the minimum-energy accumulative broadcast problem. We present a solution employing two subproblems. First, we identify the ordering in which nodes should transmit. Second, we determine the optimum power levels for that ordering. While the second subproblem can be solved by means of linear programming, the ordering subproblem is found to be NP-complete. We devise a heuristic algorithm to find a good ordering and evaluate the performance of the algorithm. Preliminary results show the performance of the heuristic algorithm is generally close to the optimum solution. Results also show a significant improvement compared to the well known BIP algorithm for constructing an energy-efficient broadcast tree.
\end{abstract}

\section{Introduction.}

In the problem of minimum-energy broadcasting in a wireless network, the objective is to broadcast data reliably to all network nodes at a fixed rate with minimum transmitted power. The problem of broadcasting in a wireless network was introduced in [13] and formulated as a minimum-cost broadcast tree problem. In a wired network, the minimum-cost broadcast tree can be found in $O\left(n^{2}\right)$ operations [8]. However, in the wireless network, this problem was shown in [6] to be NP-hard and later on, in $[1,2,7]$ to be NP-complete. The greater difficulty of the wireless broadcast problem tree stems from the "wireless multicast advantage" [13], the fact that a wireless transmission can be received by all nodes in the transmission range. Several heuristics for constructing energy-efficient broadcast trees have been proposed in literature and evaluated through simulations. The quantitative characterization of three such algorithms is given in [12].

The wireless formulation of the minimum energy broadcast problem assumes that a node can benefit from a certain transmission only if the received power is above a threshold required for reliable communication. This is a pessimistic assumption. A node for which the received power is below the required threshold, but above the receiver noise floor, can collect energy from the unreliable reception of the sent information. For example, in a Bluetooth system [9], the nominal transmitted power is $1 \mathrm{~mW}$ resulting in a transmission range of 10 meters. 
However, for a typical path exponent of $\alpha=3$, the received signal at a node within 90 meters of the transmitter is likely to be above the receiver noise floor.

Moreover, it was observed in the relay channel [3] that utilizing unreliable overheard information was essential to achieving capacity. We borrow this idea and re-examine the minimum energy broadcast problem under the assumption that nodes can exploit the energy of an unreliable reception. For the broadcast problem, a node will have multiple opportunities to receive a message as it is forwarded through the network. We refer to this strategy as accumulative broadcast.

Even in the simplest case of a single relay node, finding the maximum achievable common rate for a given set of transmit powers is, in general, an unsolved open problem. Even in the special case of the physically degraded single relay channel, key techniques employed in [3] to enable coordination of the transmissions of the source and the relay are not easily extensible to multiple node networks. In this work, we seek to employ overheard information in a large scale network. To do this, we make certain simplifying assumptions. First, our focus will be on networks in which bandwidth is plentiful relative to the transmit power available to the nodes. Second, we impose a reliable forwarding constraint that a node can forward a message only after reliably decoding that message. Although reliable forwarding is generally a suboptimal strategy, we will see that it greatly simplifies the system architecture while still allowing us to benefit from unreliable overheard information.

In the following section, we give the network model. In Section 3, we describe our approach for finding the best broadcasting solution and show that the problem is NP-complete. A heuristic algorithm and its performance is presented in Section 4. Due to space limitations, we were unable to provide proofs for most theorems in this paper.

\section{System model.}

We consider a wireless network of $N$ nodes such that from each transmitting node $k$ to each receiving node $m$, there exists an AWGN channel of bandwidth $W$ characterized by a frequency non-selective link gain $h_{m k}$. We further assume large enough bandwidth resources to enable each transmission to occur in an orthogonal channel, thus causing no interference to other transmissions. Each node has both transmitter and receiver capable of operating over all channels.

A receiver node $j$ is said to be in the transmission range of transmitter $i$ if the received power at $j$ is above a threshold that ensures the capacity of the channel from $i$ to $j$ is above the code rate of node $i$. We assume that nodes can use different power levels, which will determine transmission ranges. The nodes beyond the transmission range can exploit the fact that a message is sent through multiple hops on its way to all the nodes. Repeated transmissions act as a repetition code for all the nodes beyond the transmission range.

We view each orthogonal channel as a discrete-time Gaussian channel by representing a waveform of duration $T$ as a vector in the $n=2 W T$ dimensional space [4]. Then, during the $i$ th symbol interval of length $T$, a source node, labeled node 1, transmits a codeword (vector) $X^{n}(i)$ from a $\left(2^{n R}, n\right)$ Gaussian code that is generated according to the distribution $p\left(X^{n}\right)=\prod_{l=1}^{n} p\left(x_{l}\right)$ where $p(x) \sim N(0,1)$. Under the reliable forwarding constraint, a node $j$ is permitted to retransmit (forward) codeword $X^{n}(i)$ only after reliably decoding $X^{n}(i)$. With an appropriate set of retransmissions at appropriate power levels, eventually every node will have reliably decoded $X^{n}$. Henceforth, we drop the index $i$ and focus on the broadcast of a single codeword $X^{n}$. We will say a node is reliable once it has reliably decoded $X^{n}$. 
The constraint of reliable forwarding imposes an ordering on the network nodes. In particular, a node $m$ will decode $X^{n}$ from the transmissions of a specific set of transmitting nodes that became reliable prior to node $m$. Starting with node 1 , the source, as the first reliable node, a solution to the accumulative broadcast problem will be characterized by a reliability schedule, which specifies the order in which the nodes become reliable.

A reliability schedule $\left[n_{1}, n_{2}, n_{2}, \ldots, n_{N}\right]$ is simply a permutation of $[1,2, \ldots, N]$ that always starts with the source node $n_{1}=1$. Given a reliability schedule, it will be convenient to relabel the nodes such that the schedule is simply $[1,2, \ldots, N]$. After each node $k \in\{1, \ldots, m-1\}$ transmits codeword $X^{n}$ with power $P_{k}$, the received signal at node $m$ for each symbol $x$ in the codeword is

$$
\mathbf{y}=\mathbf{h} x+\mathbf{n},
$$

where $\mathbf{h}=\left[h_{1}, \ldots, h_{m-1}\right]^{T}$ has $k$ th element $h_{k}=\left(\sqrt{h_{m k} P_{k}}\right)$ equal to the received power corresponding to the transmission of node $k$ and $\mathbf{n}$ is is a random noise vector with covariance matrix $\mathbf{K}_{\mathbf{n}}=\sigma^{2} \mathbf{I}_{K}$. It follows that the mutual information is

$$
I(x ; \mathbf{y})=\frac{1}{2} \log _{2}\left(1+\frac{\sum_{k=1}^{m-1} h_{m k} P_{k}}{\sigma^{2}}\right) \quad \text { bits. }
$$

This is precisely the capacity of a multi-antenna system derived in [10] with $m-1$ transmitting antennas and one receiving antenna. It follows from Equation (2) that the maximal number of bits per second that can be transmitted in the system given by Equation (1) is

$$
r_{m}=W \log _{2}\left(1+\frac{\sum_{k=1}^{m-1} h_{m k} P_{k}}{N_{0} W}\right) \quad \text { bits/s, }
$$

where $N_{0}$ is the one-sided power spectral density of the noise.

Acheiving $r_{m}=\bar{r}$ implies $\sum_{k=1}^{m-1} h_{m k} P_{k} \geq \bar{P}$ where

$$
\bar{r}=W \log _{2}\left(1+\frac{\bar{P}}{N_{0} W}\right) \quad \text { bits/s. }
$$

is the fixed data rate used for broadcasting.

When the nodes are power limited and the data rate $\bar{r}$ is small relative to the channel bandwidth $W$, the system operates in the wideband regime where energy per bit is close to its minimum value and the increase in rate with power is linear:

$$
\bar{r}_{\infty}=\lim _{W \rightarrow \infty} \bar{r}=\lim _{W \rightarrow \infty} W \log _{2}\left(1+\frac{\bar{P}}{W N_{0}}\right)=\frac{\bar{P}}{N_{0} \ln 2} \quad \text { bits/s. }
$$

From the above equation, transmission of $\bar{r}_{\infty}=E_{T} /\left(N_{0} \ln 2\right)$ bits requires received energy of $E_{T}=\bar{P} T$, revealing the well known fact that for the reliable reception of one information bit, a receiver has to collect at least $E_{T}=N_{0} \ln 2$ Joules/bit. [11]. This energy can be collected at a node $m$ during one transmission interval $[0, T]$ when a transmitter $j$ is signaling with power $P=\left(N_{0} \ln 2\right) /\left(h_{m j} T\right)$. However, in the system given by Equation (1), the required energy $E_{T}$ is collected in $m-1$ repeated transmissions. In the wideband regime, Equation (3) is

$$
\lim _{W \rightarrow \infty} r_{m}=\frac{1}{N_{0} \ln 2} \sum_{k=1}^{m-1} h_{m k} P_{k} .
$$

Next we show that for the same transmitted powers, using a coding strategy other than the repetition code described above, cannot increase the rate achieved at the receiving node $m$. 
Theorem 1 For the wideband regime, fixed transmitted powers $\left\{P_{1}, \ldots, P_{N}\right\}$ and a reliability schedule $[1,2, \ldots, N]$, the maximum rate achievable from the source to node $m$ is given by Equation (2) and is achieved by the repetition coding strategy.

Proof. An upper bound to the achievable rate between the source and the destination is the maximum conditional mutual information across a minimum cut [4]. Consider the multiaccess cut in the given network that separates the destination node from the rest of the network. Let $X_{j}$ denote a symbol transmitted at node $j$ and $Y$ denote the received signal at the destination. The maximum mutual information across this cut is given by

$$
C_{\mathrm{MAC}}=I\left(X_{1}, \ldots X_{m-1} ; Y\right) \text {. }
$$

In the considered network, each orthogonal channel is assigned bandwidth $W$ and hence the mutual information above is given by the sum of rates achieved in each of the channels. For Gaussian channels,

$$
C_{\mathrm{MAC}}=W \sum_{k=1}^{m-1} \log _{2}\left(1+\frac{h_{m k} P_{k}}{N_{0} W}\right) .
$$

In the wideband regime, Equation (8) becomes

$$
C_{\mathrm{MAC}}=\lim _{W \rightarrow \infty} W \sum_{k=1}^{m-1} \log _{2}\left(1+\frac{h_{m k} P_{k}}{N_{0} W}\right)=\frac{1}{N_{0} \ln 2} \sum_{k=1}^{m-1} h_{m k} P_{k},
$$

which is precisely the rate given by (6) achieved using the repetition strategy. Since this rate is achievable, this cut is the minimum cut. No better rate can be achieved since it would violate the condition for the upper bound.

Not allowing the noisy observations to be amplified and sent at relays makes achieving the broadcast cut-set bound impossible. As shown in [5], this bound can be asymptotically achieved in a network with one source-destination pair as the number of relays goes to infinity. Again, this situation is equivalent to the multi-antenna system [10], but in this case, with one transmitting antenna and $K$ receiving antennas.

\section{Approach}

Under the constraint of reliable forwarding, an optimal solution to the minimum power accumulative broadcasting problem must specify the reliability schedule as well as the transmitter power levels used at each node. An optimal choice of the reliability schedule will result in minimum total transmitted power over the set of nodes.

From the above arguments, it follows that our problem of finding the minimum-energy accumulative broadcast differs from the minimum-energy broadcast tree $[1,2,6,7,12-14]$ in the sense that no received energy is wasted at the nodes while they collect energy unreliably. The two problems are similar in that finding the optimum solution involves the right ordering of relay nodes and determination of transmitter power levels. Defining the broadcast tree determines all the transmission levels and thus solves the problem completely; a relay that is the parent of a group of nodes in the broadcast tree transmits with the power needed to reliably reach the most disadvantaged node in the group. Hence, the arcs in the broadcast tree uniquely determine the power levels for each transmission.

In our scenario, however, the broadcast tree loses its meaning since nodes collect energy from the transmissions of many nodes. The optimum solution may require that a relay transmits with a power level different from the level precisely needed to reach a group of nodes 
reliably; the nodes can collect the rest of the needed energy from the future transmissions of other nodes. In fact, the optimum solution favors such situations because all nodes beyond the range of a certain transmission are collecting energy while they are unreliable; the more such nodes, the more efficiently the transmitted energy is being used. Therefore, if during one transmission, an unreliable node is collecting energy faster due to a good channel gain with a current transmitting node, it may be desirable for the transmitting node to stop transmitting before the collected energy at the node in favor reaches the threshold of reliable communication; that node will collect the rest of the energy needed during future transmissions of other nodes.

The next theorem shows that this approach can never do worse than the solution to the minimum-energy broadcast tree approach. In fact, accumulative broadcasting will always result in a more energy-efficient solution.

Theorem 2 The sum of transmitted powers of the minimum-energy broadcast tree is an upper bound to the sum of transmitted powers for accumulative broadcasting.

The differences in our problem from the minimum-energy broadcast tree dictate a new approach. The crucial step is finding the best reliability schedule. We call such an order a schedule. Given a schedule, we can formulate a linear program (LP) that will find the optimum solution for that schedule. Such a solution will identify those nodes that should transmit and their transmission power levels. Solving the LP for all possible schedules and taking the minimum-energy solution among all the LP solutions will result in the optimum schedule, and optimum transmission power levels. This divides the problem into two subproblems.

To define the LP for a certain schedule, we use the observation that every node selected to transmit by the optimum solution, needs to transmit only once. This fact is given by the next theorem.

Theorem 3 Given a solution to the accumulative broadcast problem consisting of a sequence of transmissions where a node $j$ is assigned to transmit $K$ times with power levels $P_{j}^{1}, \ldots P_{j}^{K}$ then there is a feasible optimum solution in which node $j$ transmits only once with power level $\sum_{k=1}^{K} P_{j}^{k}$.

Mathematically, a reliability schedule can be represented by a matrix $\mathbf{X}$ where

$$
x_{i j}= \begin{cases}1 & \text { if node } i \text { is scheduled to transmit after node } j \\ 0 & \text { otherwise }\end{cases}
$$

Each $x_{i j}$ is an indicator that a node $i$ collects energy from a transmission by node $j$. Note that $x_{i i}=0$, for all $i$ and $x_{j i}=1-x_{i j}$. Given $\mathbf{X}$ we define a gain matrix for that schedule $\mathbf{H}(\mathbf{X})$. Each element $(i, j)$ in $\mathbf{H}(\mathbf{X})$ is given as $h_{i j} x_{i j}$. In terms of the vector $\mathbf{p}$ of transmitted powers, the LP for that schedule is

$$
\begin{aligned}
& \rho(\mathbf{X})= \min \mathbf{1}^{T} \mathbf{p} \\
& \text { subject to } \mathbf{H}(\mathbf{X}) \mathbf{p} \geq \mathbf{1} \bar{P}, \\
& \mathbf{p} \geq \mathbf{0} .
\end{aligned}
$$

The inequality $\mathbf{H}(\mathbf{X}) \mathbf{p} \geq \mathbf{1} \bar{P}$ contains $N-1$ constraints requiring that the received power at all the nodes but the source is above the required threshold $\bar{P}$. Given a schedule $\mathbf{X}$, we will use $\mathbf{p}^{*}(\mathbf{X})$ to denote a power vector $\mathbf{p}$ that achieves total transmitted power $\rho(\mathbf{X})$.

In a schedule, all $N$ nodes are given a chance to transmit the data (since $p_{j}$ can be greater than 0 for every node) and only the order of transmission is different for different schedules. 


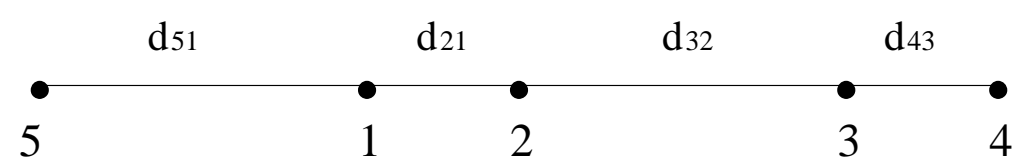

Figure 1: A five node network example.

Every schedule is a different ordered sequence of $N$ nodes. Since the source always transmits first, there are $(N-1)$ ! schedules corresponding to the number of permutations of $N-1$ elements. Thus, out of $N^{(N-2)}$ broadcast trees, we consider a subset of $(N-1)$ ! schedules. The reason is that, if the best solution is that only a subset of nodes should be transmitting, the appropriate LP will find that solution by setting appropriate powers to zero. In general, however, the problem of finding a best schedule is intractable.

Theorem 4 The existence of a schedule $\mathbf{X}$ such that $\rho(\mathbf{X}) \leq B$ is an NP-complete problem.

In the following, we describe scheduling heuristics.

\section{Scheduling Heuristics}

We observe that we can restrict ourselves to scheduling nodes in an order in which they can become reliable one at a time. When a node $j$ is scheduled to be the next node in a schedule after a set of nodes $S$, then a transmission from that set has to make node $j$ reliable. If the power that is needed to reach node $j$ is enough to reach another unreliable node $i$ as well, than we could have done better by assigning node $i$ for transmission before node $j$. This is because $i$ cannot benefit from a transmission from node $j$ (since it is made reliable before $j$ ) but $j$ might benefit from a transmission from $i$. This reasoning will be used in the proposed heuristic algorithm.

The next theorem shows how the solution of a LP for a certain schedule gives information about the subsequent schedule to be considered.

Theorem 5 If the solution $\mathbf{p}^{*}=\mathrm{p}^{*}(\mathrm{X})$ of the LP (11) has a subset of nodes $I$, such that $p_{i}^{*}=0$ for $i \in I$, then $\rho(\hat{\mathbf{X}}) \leq \rho(\mathbf{X})$ for a new schedule $\hat{\mathbf{X}}$ that assigns nodes in I to transmit last.

We illustrate our approach on an example network with 5 nodes on a line as shown in Figure 1. For every two nodes $j$ and $k$, the link gain is calculated from $h_{j k}=d_{j k}^{-3}$ for the distances given in Figure 1. Let node 1 be a source. The number of possible spanning trees is 125 . By Theorem 3, $(N-1)$ ! = 24 schedules need to be considered given by all the possible permutations of all the nodes except the source who always transmits first. Two observations help reduce the number of schedules: Since the closest neighbor to the source is node 2, this node will become reliable first regardless of a schedule. Following the reasoning from above, all the considered schedules should assign this node for a possible transmission after the source. For the same reason node 3 should always be scheduled to transmit before node 4 , thus giving node 4 the chance to collect energy from the transmission of node 3 . This leaves only three possible schedules to be considered:

$$
\mathbf{s}_{1}=[1,2,3,4,5], \quad \mathbf{s}_{2}=[1,2,3,5,4], \quad \mathbf{s}_{3}=[1,2,5,3,4] .
$$




$$
\begin{aligned}
& \mathbf{s}=[1] ; \quad \mathbf{p}=0 \\
& \text { while }(|S|<N) \text { do } \\
& k=\arg \max _{i \in S} \sum_{j \in U} h_{j i} ; \\
& j=\arg \min _{m \in U}\left(\bar{P}-\sum_{i \in S} h_{m i} p_{i}\right) / h_{m k} ; \\
& n=I_{k}(\mathbf{s}) ; \\
& \text { if } \quad\left(n \neq|S| \text { and } p_{k} \neq 0\right) \\
& p_{k} \leftarrow p_{k}+\left(\bar{P}-\sum_{i \in S} h_{j i} p_{i}\right) / h_{j k} ; \\
& \text { for } i=n+1:|S| \text { do } p_{s_{i}}=0 \text { end; } \\
& \quad \mathbf{s} \leftarrow\left[s_{1}, \ldots, s_{n}\right] ; \\
& \text { do } \quad l=\arg \max _{m \in U} \sum_{i \in S} h_{m i} p_{i} ; \\
& \quad \text { if }\left(\sum_{i \in S} h_{l i} p_{i} \geq \bar{P}\right) \text { s } \leftarrow[\mathbf{s}, l] ; \\
& \text { while }\left(\sum_{i \in S} h_{l i} p_{i} \geq \bar{P}\right) \\
& \text { else } \quad p_{k} \leftarrow p_{k}+\left(\bar{P}-\sum_{i \in S} h_{j i} p_{i}\right) / h_{j k} ; \\
& \quad \mathbf{s} \leftarrow[\mathbf{s}, j] \\
& \text { end } ; \\
& \text { end }
\end{aligned}
$$

Given a partial schedule $\mathbf{s}, S$ is the unordered set of nodes in $\mathbf{s}$ and its complement $U$ is the set of unreliable nodes. The cardinality of $S$ is given by $|S|$. In addition, $I_{k}(\mathbf{s})$ returns the position of node $k$ in the schedule $\mathbf{s}$. That is, if $s_{n}=k$, then $I_{k}(\mathbf{s})=n$.

Figure 2: Greedy Filling Heuristic Algorithm

We consider schedule $\mathbf{S}_{1}$ first. The LP for that schedule is

$$
\begin{gathered}
\min p_{1}+p_{2}+p_{3}+p_{4} \\
{\left[\begin{array}{llll}
h_{21} & & & \\
h_{31} & h_{32} & & \\
h_{41} & h_{42} & h_{43} & \\
h_{51} & h_{52} & h_{53} & h_{54}
\end{array}\right]\left[\begin{array}{l}
p_{1} \\
p_{2} \\
p_{3} \\
p_{4}
\end{array}\right] \geq \mathbf{1} \bar{P}} \\
\quad p_{1}, p_{2}, p_{3}, p_{4} \geq \mathbf{0}
\end{gathered}
$$

Solving the LP we get the optimum power levels for this schedule. In particular, the solution finds that $p_{4}=0$. Therefore, although node 4 was given a chance to transmit as soon as possible, its transmission is not needed; by theorem 5 we can move node 4 to the end of the schedule. In both schedules $\mathbf{s}_{2}$ and $\mathbf{s}_{3}$ node 4 is scheduled to be the last node in the schedule. Hence, we consider both $\mathbf{s}_{2}$ and $\mathbf{s}_{3}$. By solving the LP for those two schedules, and comparing the minimum energy solution for three schedules, we get the optimum solution. The solution gives nodes 1, 2 and 3 as the transmitting nodes. The power level at node 1 is higher than needed to reach node 2 but less than needed to reach node 5 ; the rest node 5 collects from later transmission at nodes 2 and 3. The transmitted power at node 2 is enough to reach node 3 given what it collected from $p_{1}$. Node 3 transmits to 4 such that enough energy from three transmissions is collected at node 4 to meet the threshold.

Because of the complexity of the problem of finding the best schedule, we now propose a heuristic algorithm that finds a good schedule. Once the schedule is determined, the LP for that schedule is solved to find the optimum power levels. We evaluate the performance of the algorithm through simulation and compare its power efficiency to the optimum solution as well as to the performance of BIP, the heuristic algorithm proposed in [13]. 


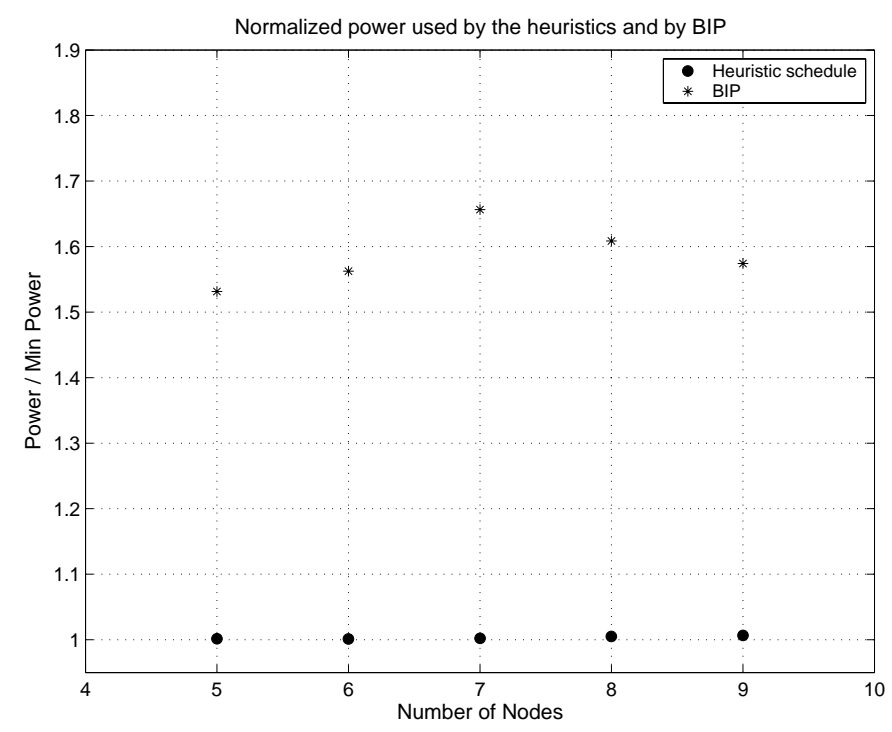

Figure 3: Normalized power used for broadcasting.

The algorithm pseudocode is given in Figure 2. The algorithm starts with a partial reliability schedule $\mathbf{s}=[1]$ that contains only the source. In general, given $\mathbf{s}=\left[s_{1}, \ldots, s_{n}\right]$, if $s_{l}=k$, then node $k$ is the $l$ th node in the schedule. Given a partial schedule $\mathbf{s}$, we find the reliable node $k$ that maximizes the fill rate of the unreliable set. By Theorem 3, node $k$ should transmit only once. Therefore, we check to make sure that $k$ has not yet transmitted, or if it did, $k$ was the last reliable node to transmit. In this case, we increase $p_{k}$ by the power needed to add one more node, node $j$, to the reliable set and we simply append node $j$ to the schedule. Otherwise, if $k$ is not the last reliable node to transmit and $p_{k} \neq 0$, then $k$ has been chosen to transmit earlier in the schedule. By Theorem 3, $k$ should have transmitted at that earlier time with a higher power level, making more of its neighbors reliable at the same step. Thus we reset the schedule after node $k$ in the following way. All transmitting nodes that followed node $k$ in the schedule have their powers set to zero and we truncate the schedule after node $k$. However, those nodes that are made reliable by transmissions of the truncated schedule are appended to the schedule in order of decreasing total received power. Once the schedule is complete, the LP is solved to find the optimum power levels for that schedule.

We evaluated the performance of the algorithm and compared it to the optimal solution as well as to the performance of BIP. Networks with a small number $(5-10)$ of randomly positioned nodes were generated. We also compared the performance of two heuristics for more dense networks. The transmitted power was attenuated as $r^{\alpha}$ for propagation exponent $\alpha=2$. The received power threshold was chosen to be $\bar{P}=1$. The results were based on the performance of 100 randomly chosen networks. When comparison with the optimum solution was performed, the performance metric used was the normalized total power in the network. In each simulation run, the power used when a heuristic algorithm was employed was normalized by the power used in the optimum solution.

Results are shown in Figure 3 as a function of the number of network nodes. Results show the heuristic algorithm performance very close to the optimum. This is a desirable and important characteristic, given the complexity of finding the optimum solution. The simulation result also show the noticeable improvement in the power saving in our approach compared to power used when the minimum-energy broadcast tree approach is used and the broadcast tree is found using BIP. 


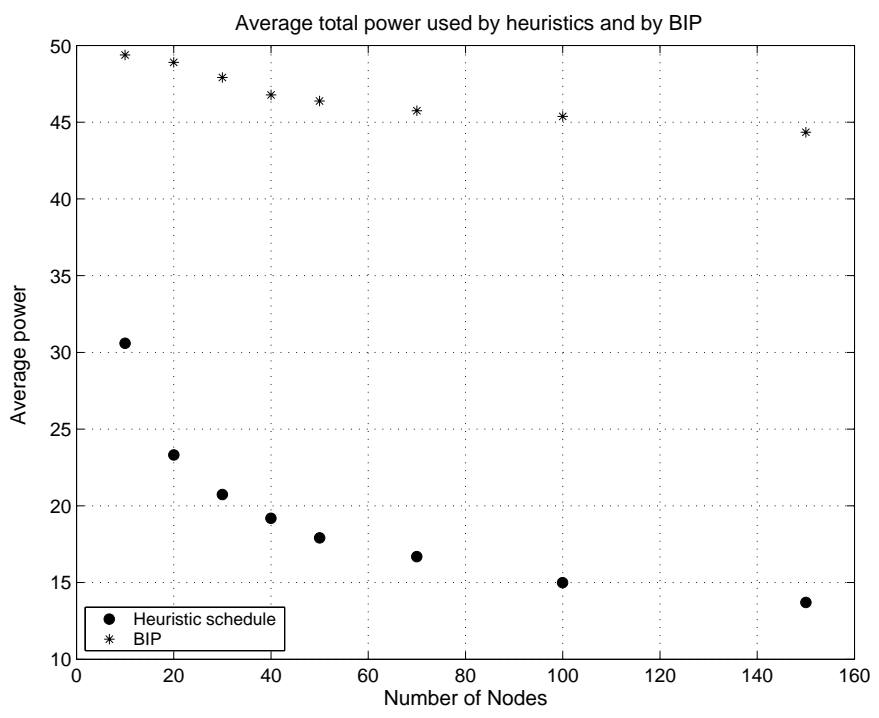

Figure 4: Average total power used for broadcasting.

For the networks with a larger number of nodes, performance results for the accumulative broadcast heuristics and BIP are shown in Figure 4. The metric used in this case was the average total power used for broadcasting. We observe that total power decreases with the number of nodes due to the increased number of shorter hops. The decrease in the case of the accumulative broadcast is steeper since the increased number of transmissions allows for more energy to be collected. Hence the relative improvement over BIP increases with the number of network nodes.

\section{Conclusion}

In this paper we address the minimum-energy broadcast problem. To increase the energy efficiency, we realize that a lot of energy is radiated into the network in each transmission and thus, we allow nodes outside of the transmission range to collect the energy of the unreliably received signal. A node will have multiple opportunities to receive reliably the message by collecting energy while the message is forwarded through a network. We demonstrate that finding the optimum solution to this problem is complex and therefore, propose a heuristic algorithm that has energy-efficiency close to optimal and can still provide energy-saving compared to broadcasting that doesn't collect energy from unreliable transmission.

\section{References}

[1] A. Ahluwalia, E. Modiano, and L. Shu. On the complexity and distributed construction of energy-efficient broadcast trees in static ad hoc wireless networks. In Proc. of Conf. on Information Science and Systems, March 2002.

[2] M. Cagalj, J. Hubaux, and C. Enz. Minimum-energy broadcast in all-wireless networks: Np-completeness and distribution issues. In Proc. of Mobicom'02, 2002.

[3] T. Cover and A. El Gamal. Capacity theorems for the relay channel. IEEE Trans. on Information Theory, 25(5):572-584, September 1979. 
[4] T. Cover and J. Thomas. Elements of Information Theory. John Wiley Sons, Inc., 1991.

[5] M. Gastpar and M. Vetterli. On the capacity of wireless networks: The relay case. In Proc. of INFOCOM'02, June 2002.

[6] F. Li and I. Nikolaidis. On minimum-energy broadcasting in all-wireless networks. In Proc. of Local Computer Networks (LCN’01), 2001.

[7] W. Liang. Constructing minimum-energy broadcast trees in wireless ad hoc networks. In Proc. of International Symposium on Mobile Ad Hoc Networking and Computing (MobiHoc'02), June 2002.

[8] C.H. Papadimitriou and K. Steiglitz. Combinatorial Optimization: Algorithms and Complexity. Prentice Hall, Englewood Cliffs, NJ, 1982.

[9] SIG. Bluetooth specification version 1.0b. Tech. Spec., 1999.

[10] E. Telatar. Capacity of multi-antenna gaussian channels. In Europ. Trans. Telecommunications, November 1997.

[11] S. Verdú. Spectral efficiency in the wideband regime. IEEE Trans. on Information Theory, 48(6):1319-1343, June 2002.

[12] P-J. Wan, G. Calinescu, X-Y. Li, and O. Frieder. Minimum-energy broadcast routing in static ad hoc wireless networks. In Proc. of INFOCOM'02, April 2001.

[13] J.E. Wieselthier, G.D. Nguyen, and A. Ephremides. On the construction of energyefficient broadcast and multicast trees in wireless networks. In Proc. of Infocom 2000, 2000 .

[14] J.E. Wieselthier, G.D. Nguyen, and A. Ephremides. Algorithms for energy-efficient multicasting in static ad-hoc wireless network. Mobile Networks and Applications, pages 251-263, 2001. 